Purdue University Purdue e-Pubs

2010

\title{
Resistance network-based thermal conductivity model for metal foams
}

\author{
Karthik K. Bodla \\ Purdue University - Main Campus \\ Jayathi Y. Murthy \\ Purdue University - Main Campus \\ S V. Garimella \\ Purdue University, sureshg@purdue.edu
}

Follow this and additional works at: http:// docs.lib.purdue.edu/coolingpubs

Bodla, Karthik K.; Murthy, Jayathi Y.; and Garimella, S V., "Resistance network-based thermal conductivity model for metal foams" (2010). CTRC Research Publications. Paper 141.

http://dx.doi.org/doi:10.1016/j.commatsci.2010.09.026

This document has been made available through Purdue e-Pubs, a service of the Purdue University Libraries. Please contact epubs@purdue.edu for additional information. 
1

\title{
Resistance Network-Based Thermal Conductivity Model for Metal
}

\section{Foams}

\author{
Karthik K. Bodla, Jayathi Y. Murthy and Suresh V. Garimella' \\ School of Mechanical Engineering, Purdue University \\ West Lafayette, Indiana 47907-2088
}

\begin{abstract}
A network model for the estimation of effective thermal conductivity of open-celled metal foams is presented. A nodal network representation of three aluminum foam samples from DUOCEL - 10 ppi, 20 ppi and 40 ppi - is constructed out of X-ray microtomography data obtained by Computed Tomography (CT) scanning of the samples using a commercial CT scanner. Image processing and 3D skeletonization are performed with commercially available image processing software. The effective thermal conductivity is estimated through a $1 \mathrm{D}$ conduction model, representing individual ligaments as an effective thermal resistance using the topological information from the scan data. The effective thermal conductivity data thus obtained are compared with the Lemlich theory and other pore-based models. Further, microstructural characterization of foam features - pore size, ligament thickness, ligament length and pore shapes - is performed. All the three foam samples are observed to have similar pore shapes and volumetric porosity, while the other features scale with the pore size. For a given porosity the computed permeability is found to scale as the square of the pore diameter, as also noted by previous researchers.
\end{abstract}

Keywords: metal foams, X-ray microtomography, effective thermal conductivity, skeletonization, 1D resistance network model, microstructure, permeability

\footnotetext{
${ }^{1}$ Corresponding author, Email: sureshg@purdue.edu, Tel: 765-494-5621
} 


\section{Introduction}

Open-celled metal foams, initially developed for structural applications, have been shown to have desirable thermal properties such as a high heat transfer coefficient and a high surface area-to-volume ratio, enabling them to be used as effective cooling solutions in many thermal problems [1-3]. Additional desirable features include weight and volume reduction [4].

Much of the earlier thermal analysis of open-celled metal foams has been largely experimental. Calmidi and Mahajan [5] performed thermal conductivity measurements of fibrous metal foams with air and water as the interstitial fluids, and proposed a model with an adjustable free parameter to match the experimental results obtained. Paek et al. [6] performed both thermal conductivity and permeability measurements for highly porous aluminum foams with air as the interstitial fluid.

Modeling efforts have traditionally employed one of two approaches: a) a macroscopic or semiempirical approach, such as in [5] and [7], and b) a microscopic or pore-based approach [8]. Semiempirical approaches employ volume-averaged equations to describe flow and heat transfer characteristics. On the other hand, the microscopic approach involves pore-based calculations, employing idealized periodic cell representations of the cellular metal foam geometry. Geometries such as cubic unit cells with square cylinders [9] and minimal surface area-to-volume ratio-based BCC structures [8] have been widely employed in the literature.

X-ray microtomography (XMT) offers a relatively new modeling approach with which the randomness of the porous medium is accurately captured. The advent of superior algorithms and submicron scale resolutions have enabled the employment of XMT for visualizing intricate details of materials such as random porous media. Fiedler et al. [10] employed a lattice Monte Carlo approach directly on the XMT data to perform transient thermal conduction measurements. Laschet et al. [11] performed thermal conductivity predictions of Inconel foam samples employing a homogenization approach by generating finite element (FE) models of scanned foam samples. In earlier work [12], we performed detailed finite volume CFD simulations of the flow and heat transfer properties of aluminum 
foams by generating computational meshes based on computed tomography (CT) data. Other nonconventional approaches include the use of Laguerre tessellations generated by random sphere packings [13].

Network-based analysis may be used for analytically calculating many of the characteristics of random materials, such as permeability and effective thermal conductivity. Some of the earlier models for predicting the effective thermal conductivity (for example, [14]) are based on series and parallel combinations of thermal resistances. Fatt [15] employed the analogy between electrical conduction and fluid flow (Darcy's law) for estimation of permeability of porous media. Raoof and Hassanizadeh [16] developed a new multi-directional pore network representation of porous media based on a cubic lattice network, and compared the co-ordination number with that obtained through CT. In the present work, we develop a network model for calculating effective thermal conductivity, employing the analogy between thermal and electrical conduction. The data pertaining to the foam microstructure generated through XMT is employed for developing the proposed network model. This approach may also be extended to other types of porous media, and for estimating properties such as permeability by exploiting the analogy between fluid flow and electrical conduction.

\section{Materials under consideration}

The materials studied in this work are highly porous aluminum foam samples with porosity (void fraction) in the range of 90-92\%. Aluminum foams are a class of cellular metals with novel thermal and thermo-mechanical properties such as high thermal conductivity, high permeability to fluids and low specific weight [17]. Metal foams are manufactured in a number of ways ranging from direct foaming to vapor deposition. Banhart [18] provided an excellent review of the various manufacturing processes employed in foaming metals. Conventional techniques such as direct foaming of molten metal by gas injection tend to produce closed-cell morphologies, while open-celled porous metals may be manufactured by investment casting with polymer foams as noted in [18]. In this process, an open-celled polymer foam which is produced conventionally, and is thereby reticulated, is the starting material. It is 
first filled with a slurry of heat-resistant material such as mullite and then cured. The polymer foam is subsequently removed by thermal treatment to produce voids replicating the original foam, which can then be filled with molten metal to produce an open-celled metal foam. The foaming company, ERG, employs this method to produce aluminum foams under the trade name DUOCEL [19]. Three DUOCEL foam samples of varying pore size - $10 \mathrm{ppi}, 20 \mathrm{ppi}$ and $40 \mathrm{ppi}$ - are considered in this work. Thermal and mechanical properties of DUOCEL foams may be found in [19].

\section{Sample preparation}

Commercially available XMT scanners feature a trade-off between the scan resolution and the size of the sample being scanned. In this work, foam samples of size $10 \mathrm{~mm}$ x $12.7 \mathrm{~mm}$ x $38.1 \mathrm{~mm}$ are cut from original foam slabs of size $12.7 \mathrm{~mm}$ x $38.1 \mathrm{~mm}$ x $88.9 \mathrm{~mm}$ using electric discharge machining (EDM), to ensure that the cuts are clean and that the cut samples are not distorted. The samples are then imaged using axial $\mu$-CT at 20 micron resolution employing a commercial X-ray scanner- $\mu \mathrm{CT} 40$ (SCANCO MEDICAL), with the axis being along the longest dimension. The resolution is selected such that the ligaments are properly reconstructed in the image reconstruction step and that other microscale features, such as the pore orientation and shape, are well-represented. The scanner software generates a 2D stack of images corresponding to the $3 \mathrm{D}$ object scanned, which can be later analyzed after surface/volume reconstruction.

\section{Image processing and skeletonization}

The images obtained from the scan data suffer from a number of artifacts such as noise and blurriness and hence the images must be processed further before skeletonization. The image processing and material identification steps are described only briefly in this paper; a detailed discussion may be found in $[20]$. 


\subsection{Image processing}

The commercially available image processing package AMIRA is used in this work for image processing. The scan region (foam and pore) is first separated from the background by cropping an appropriate region of interest. Resizing is performed in all three dimensions to match the size of the original sample. A Gaussian filter with default parameters as described in [20] is then applied to reduce noise in the images. The scan data at this stage consist of a large number of pixels which increases the demand on memory of the image processing package when used for advanced processing such as skeletonization. Hence, the images are first carefully down-sampled such that the foam ligaments are still well-represented. These image processing steps are illustrated in Fig. 1.

\subsection{Image segmentation and skeletonization}

XMT, based on the differential absorptivity of different materials to X-rays, generates images which reflect these differences in terms of grayscale values. For our samples, two different materials - metal and air - may be identified by examining the scan data. Metal, being dense, blocks X-rays, while air lets most of the X-rays to pass through. This difference is reflected in the corresponding images where the brighter region corresponds to the metal and the darker region to the surrounding pore. However, the transition is not smooth, and therefore, detection based on a threshold value selected from the image histogram data is needed. The threshold value is selected so that the porosity of the reconstructed sample is the same as that of the original scanned sample. A processed image and the corresponding segmented image are shown in Fig. 2, along with the image histogram.

The data thus segmented correspond to the metal part of the original foam sample, which can now be used to generate an image skeleton. An image skeleton or medial axis of an image is a skeletal remnant that largely preserves the extent and connectivity of the original region while discarding most of the original foreground pixels. Various definitions for the skeletons have been proposed in the literature. One of the first [21] was based on a "grass-fire" model, i.e., a moving wave-front generated by an inward motion of an outline curve with constant speed along a normal vector at every point on the curve. The 
skeleton is then defined as the set of points at which the wavefront crosses itself. It is widely employed for data reduction purposes in the fields of computer vision, medicine and pattern recognition. Image skeletons can be used for other purposes as well, such as tracking the fluid flow path in porous media and tracking the flow path of blood in blood vessels [22]. Here, the skeletonization is performed using default thinning algorithms (as described, for example, in [23]) on the segmented image data employing the commercial software AMIRA, which generates a graph-like representation of the skeleton. The skeleton for our foam sample has two essential entities - nodes and ligaments. Nodes are generated at the points where there is more than one ligament branching out; ligaments are the branches joining these nodes. A 10 ppi foam sample along with a nodal network representation of its skeleton are shown in Fig. 3 . The skeleton at this stage may consist of a number of other graphs corresponding to the 'hanging' ligaments or 'islands' in the scan data, in addition to the main graph representing the chunk of metal foam. These can be seen in Fig. 3 b) as loose unconnected ligaments, and are removed to preserve only the connected ligaments making up the chunk of the foam. In the present work, we propose the idea of using this image skeleton to estimate the effective thermal conductivity of a porous medium as described in section 5 below.

\section{Reduced-order network model}

An important property governing the utility of a metal foam sample for efficient heat transfer is its effective thermal conductivity, $k_{\text {eff, }}$ which, for a foam sample of area of cross-section $A$ is calculated as:

$$
k_{e f f}=\frac{-\int \mathbf{J} \cdot d \mathbf{A}}{\left(\frac{\partial T}{\partial x_{i}}\right) A}
$$

It is to be noted here that the effective thermal conductivity is calculated by considering conduction through both the metal and the pore regions, assuming the pore region to be saturated with an interstitial fluid such as air or water, i.e., $A$ is the total area of cross-section including both the metal and pore regions and the temperature gradient is the imposed temperature gradient. Also, $J$ in Eq. (1) is the heat flux 
vector. It may be observed from the experimental measurements of [5] and [6] that aluminum foam samples employing common fluids having a thermal conductivity 2-3 orders of magnitude lower than aluminum itself have an effective thermal conductivity which is only a weak function of interstitial fluid properties. Moreover, most commercial applications of metal foams use common fluids such as air or water as the interstitial fluid. For such applications, approximate but fast computations may be performed employing the network model proposed here, where we neglect conduction through the fluid altogether and represent the foam structure as 1D ligaments with insulated surfaces, as described in section 5.1 below.

\section{1 $1 D$ resistance network representation}

The metal foam samples considered here have ligaments with a length to diameter ratio of approximately 5. Steady-state thermal conduction through these samples may therefore be estimated by representing individual ligaments as effective 1D thermal resistance elements, although the entire network is actually three-dimensional. A 10 ppi image skeleton along with the thermal resistance network for a part of the foam sample is shown in Fig. 3 b) and c), respectively.

\subsection{Thermal conductivity model}

The thermal resistance of a cylindrical tube of area of cross-section $A_{\text {cross }}$ and thermal conductivity, $k$, for conduction along the axial direction, is given by [24]:

$$
R=\frac{L}{k A_{\text {cross }}}
$$

Representing individual ligaments of the foam samples as cylindrical resistance elements of this nature, we build a thermal resistance network consisting of a series of nodes joined by these resistance elements. At steady state, the heat flow rate through a resistance element, with temperature difference $\Delta T$ across its ends, is given by: 


$$
q=\frac{\Delta T}{R}
$$

Using Eq. (3), heat balance at any node i, joined by neighbors $\mathrm{j}(\mathrm{j}=1,2 . . \mathrm{N}, \mathrm{N}$ varying at each node), gives after rearrangement:

$$
T_{i}\left(-\sum_{j=1}^{N} \frac{1}{R_{i, j}}\right)+\sum_{j=1}^{N} \frac{T_{j}}{R_{i, j}}=0
$$

Here, $R_{i, j}$ is the thermal resistance for the ligament joining nodes $\mathrm{i}$ and $\mathrm{j}$, calculated using Eq. (2), employing topological information from the image skeleton. Eq. (4) implies that conduction - a diffusion phenomenon - leads to the temperature of a node being a weighted average of the temperatures of its neighboring nodes, the weighting function being the inverse of resistance of the link joining each node to its neighbor. That is, if the resistance of a ligament joining a node and one of its neighboring nodes is significantly lower than that of the ligaments joining other neighboring nodes, that particular neighbor has the highest influence on the node temperature and vice-versa.

Developing such equations for every node of the foam sample, a set of linear equations may be obtained, which can be cast into the following matrix form:

$$
[\mathbf{M}]_{n \times n}[\mathbf{T}]_{n \times 1}=[\mathbf{b}]_{n \times 1}
$$

The subscripts indicate the size of the matrices, with $\mathrm{n}$ being the total number of nodes. $\mathbf{M}$ is the coefficient matrix, $\mathbf{T}$ is the temperature matrix and $\mathbf{b}$ is the residual matrix. The matrix $\mathbf{M}$ is sparse. Similarly, the majority of the elements in $\mathbf{b}$ are zero. Non-zero entries in $\mathbf{b}$ correspond to boundary nodes with prescribed temperature, the equations for which are given by:

$$
T_{i, b d y}=T_{s e t}
$$

The equation set Eq. (5) can then be inverted to obtain temperature values at each node. By calculating the total heat flow rate $q$ entering (leaving) the boundary nodes as, 


$$
q_{t o t}=\sum_{i=1}^{n_{b d y}} q_{i, b d y}
$$

the effective thermal resistance of the whole foam sample may be obtained. It is to be noted that in Eq. (7) above, the summation is performed either on the hot or on the cold boundary; correspondingly, $n_{b d y}$ is the number of nodes on the hot or cold boundary. $q_{i, b d y}$ is calculated using Eq. (3). Knowing $q_{t o t}$, the effective thermal conductivity, $k_{\text {eff }}$, is then calculated as:

$$
k_{e f f}=\frac{q_{t o t} l}{A(\Delta T)_{s e t}}
$$

$l$ is the distance between hot and cold ends and $(\Delta T)_{\text {set }}$ is the temperature difference imposed on the foam sample. In Eq. (8), $A$ is the area of cross-section of the entire foam sample, i.e., it includes the area of both the pore and metal parts. In this work, the entire model is implemented in the commercial computing toolkit MATLAB. The entire computation - setting up of the matrix, matrix inversion and calculation of $k_{\text {eff }}-$ on a machine with dual Xeon processors and 3 GB of RAM took approximately 5 min.

The skeletonization algorithm in AMIRA reports the thickness of each ligament as the best-fit circle diameter, which means that the present foam samples having non-circular cross-sections are represented as cylindrical fibers in the AMIRA skeleton. When the reported thickness is directly employed, it is observed that the porosity of the sample is significantly higher (correspondingly, the metal volume fraction is significantly lower) than that of the original foam sample. A smaller cross-section implies an increased thermal resistance and correspondingly decreased effective thermal conductivity. Due to this feature in AMIRA, the reported thickness is corrected so as to match the original porosity. A single correction factor for the area of cross-section, $\triangle A$, is employed for all the metal ligaments according to:

$$
\Delta A=\frac{V_{\text {metal }, \text { actual }}-V_{\text {metal,skeleton }}}{l_{\text {skeleton }}}
$$

$l_{\text {skeleton }}$ is the length of all the segments summed together, as reported by the AMIRA skeleton. $V_{\text {metal, actual }}$ and $V_{\text {metal, skeleton }}$ are the actual and reported metal volume fractions, respectively. 


\subsection{Boundary conditions}

Analytically, the effective thermal conductivity may be estimated by employing either constant temperature boundary conditions and backing out the heat flux, or by imposing constant heat flux boundary conditions (imposing equal and opposite heat flow rates at the opposite boundaries) and backing out the temperatures at the boundaries. In this work we employ the former approach. But, unlike in an analytical solution, boundaries cannot be clearly identified in our case. This can be seen from Fig. 3, which shows a series of 'loose' nodes, corresponding to the outer boundaries, all of which do not fall on a single plane, as would be required for imposing boundary conditions. We therefore identify a thin zone on either side (along the direction in which conductivity is being estimated) and all the nodes falling inside these zones are assigned the boundary temperature. The length, $l$, used in Eq. (8) is then the minimum distance between the hot and cold boundary zones, as shown in Fig. 4 c). The thickness of the boundary zones is varied and it is observed that the randomness in the porous medium ensures that the effective thermal conductivity is independent of the boundary zone thickness. A variation of smaller than $3 \%$ in effective thermal conductivity is observed by varying the thickness of the boundary zone from 0.1 to 0.4 times the total length of the sample in the direction of the temperature gradient. Therefore, all the results reported in this work correspond to the thickness of the boundary zones being 0.2 times the total domain length in the conducting direction, unless otherwise stated.

For conduction along a particular direction, all the lateral boundaries (the boundaries parallel to the direction of the imposed temperature gradient) are insulated; in our model, this is accomplished by insulating all the nodes falling on the lateral boundaries. This is automatically implemented using Eq. (4), which is derived through energy balance.

\section{Microstructural characterization}

Open-celled metal foams have a unique microstructure, which directly influences the thermomechanical and thermal properties exhibited. Various authors have employed different representations of the metal foam microstructure for the computational estimation of these physical properties. For example, 
simple cubic unit cells consisting of slender circular cylinders were employed in [21], while a tetrakaidecahendron model was used in [8] based on mathematical arguments pertaining to the minimal surface area-to-volume ratio which must be satisfied for energy minimization during foam formation. It may be further observed from [8] and [25] that the results are different for different microstructures.

In the present work, we perform a thorough characterization of various features of the metal foams, both pertaining to the pores and the metal structure. The pore-side analysis is performed using the commercial package AVIZO, employing a set of quantification and granulometry tools ([26]). As a brief description, the images are first segmented using a threshold value as described in section 4.2, after which a watershed algorithm is employed to identify individual pores. Analysis tools in the package are then employed to determine features such as effective pore diameter and surface area of the individual pores. Further details pertaining to pore granulometry of scan data may be found in [26]. The information corresponding to the metal part of the foam is obtained from the image skeleton, constructed from the scan data using AMIRA, as described in section 4.2.

\section{Results and discussion}

This section describes the results obtained for the effective thermal conductivity with the network model developed here. The results are compared with experimental and numerical data available in the literature. The microstructural features of the metal foam samples - distributions of ligament length, ligament area of cross-section, effective pore diameter and the co-ordination number of nodes - are described for each foam sample. The three foam samples are then analyzed for their structural similarity, in terms of the sphericity of pores and non-dimensional ligament features, non-dimensionalized with corresponding effective pore diameters. Finally, the permeability data from our previous study, performed on the same foam samples [12], is compared with permeability and pore size data from the literature. 


\subsection{Effective thermal conductivity}

The thermal conductivity reported in this study is averaged along the three co-ordinate directions. The thermal conductivity values were different along different directions for the same foam sample, with a spread of as much as $15 \%$. This difference is attributed to the small sample sizes leading to a limited number of pores being included in each direction - 30-50 along the longest direction and 3-6 along the smallest direction, depending on the foam sample. Limitations posed by the scanner (as described in section 3) precluded the use of larger sample sizes. The effective thermal conductivity values obtained with the network model are shown in Table 1, with air as the interstitial fluid. Also shown are values obtained through detailed CFD simulations on samples that were half the size along the largest dimension reproduced from our prior work [12].

Fig. 4 and Fig. 5 show the temperature contours obtained with the present model and our previous work [12], respectively. Fig. 6 shows the predicted effective thermal conductivity values for the three foam samples considered in this work, along with the effective thermal conductivity data from literature, with air as the interstitial fluid. The present data are compared with experimental data [5], semi-empirical models [7], pore-based models and a detailed CFD analysis performed on the XMT data [12]. Further, Fig. 6 shows the results obtained with the Lemlich theory [27], which predicts the effective thermal conductivity as a function of thermal conductivity of the metal ligaments $\left(k_{s}\right)$ and porosity $\left(\varepsilon_{v}\right)$, by employing an analogy between thermal conduction and electrical conduction using:

$$
k_{e f f}=k_{s} \frac{\left(1-\varepsilon_{v}\right)}{3}
$$

It can be seen from Fig. 6 that the results are very sensitive to porosity and that analytical models such as the Lemlich theory (Eq. (10)) predict the trends reasonably well. However, it was shown in [8] that the Lemlich theory deviates significantly from the experimentally observed data for the case of high thermal conductivity interstitial fluids. 
It may be noted that the models of Boomsma and Poulikakos [7], Calmidi et al. [5] and Bhattacharya et al. [28] employ a free parameter to match the experiments of Calmidi et al. The model of Krishnan et al. [8] is a pore-based model, where a tetrakaidecahedral representation of the metal foam pore is employed for estimation of flow and heat transfer properties without the need for a free parameter. Our previously published model [12], on the other hand, is a direct CFD computation on the as-scanned data.

Differences of the order of $15 \%$ were observed for some samples when compared with the detailed CFD simulation [12] performed on the same samples, but with half the size considered for analysis, as described previously. This difference with respect to the detailed CFD computation is mainly attributed to the approximations in the area of cross-section employed in the current model. As described in section 5.2, an area correction $\Delta A$ is employed to ensure that the solid volume is consistent with the measured porosity of the sample. The results indicate that larger sample sizes must be used in the computation to mitigate the effect of domain-size artifacts, and multiple realizations of the sample geometry are necessary to obtain statistically invariant predictions in these random media. These caveats also apply to experimental measurements. Nevertheless, the match between the predicted results and the available literature is encouraging.

\subsection{Foam microstructure}

The metal foam microstructure is analyzed in terms of pore-based features - effective pore diameter and sphericity - and metal ligament-based features - ligament length, ligament area of cross-section and node co-ordination number. With this detailed information regarding the distribution of various features pertaining to the random metal foam samples, computational models with realistic representations of metal and pore regions may be developed, which can then be employed to estimate flow and thermal transport characteristics. Using these data, larger samples with a more realistic geometric representation may also be constructed. These are at present impossible even with XMT because of the limitations on sample sizes that can be scanned with the majority of the available commercial scanners. 


\subsubsection{Pore-basedfeatures}

The effective pore diameter is an important parameter that may be employed for describing many pore-based features such as friction factor, permeability and heat transfer coefficient. After segmenting and identifying individual pores using a watershed algorithm [29], the pores are analyzed by measuring an effective pore diameter, calculated as:

$$
d_{\text {pore }}=\sqrt[3]{\frac{6 V_{p}}{\pi}}
$$

where $V_{p}$ is the volume of the pore. The identified pores in a 10 ppi foam sample are shown in Fig. 7. The distribution of effective pore diameter is shown in Fig. 8 for the three foam samples considered in this study. The average effective pore diameter and the standard deviation for each sample are shown in Table 2. The standard deviation in pore diameter is in the range of 0.25 to 0.3 times the corresponding average values. Fig. 8 further demonstrates the need for employing a distribution of pore sizes rather than a single pore size value when modeling porous media. It may also be noted that the effective pore diameter values are significantly different from the inverse-ppi (inches per pore) values which are generally employed for the pore diameter in characterizing pore-based features. The probability density function of the pore diameter for the three foam samples is fitted using a Fourier series polynomial of order 10 as follows:

$$
p\left(d_{\text {pore }}\right)=k+\sum_{i=1}^{10} a_{i} \cos \left(i d_{\text {pore }}\right)+\sum_{i=1}^{10} a_{i} \sin \left(i d_{\text {pore }}\right)
$$

The values of the coefficients $a_{i}, b_{i}$ and the constant $k$ may be found in Table 3 along with the range over which this expression is applicable.

Another parameter of interest is the sphericity of the pores, which is a measure of the extent to which an object is spherical. The minimum surface area for a 3D object of given volume corresponds to a spherical shape. By measuring the surface area of pores and comparing it with the surface area of a fitted sphere, the sphericity $\Psi$ may be defined as: 

each other, implying that the pore shapes are essentially similar for the three, which is a consequence of the foaming process employed by ERG, as also described in [18]. The deviation of the sphericity value from that of a sphere (which has a value of 1) indicates that non-spherical pores must be employed in modeling metal foams, in contrast to the spherical pores conventionally used.

It is also interesting to consider the permeability values for the three foam samples computed previously [12]. A normalized permeability is defined as:

$$
K^{*}=\frac{K}{d_{\text {pore }}^{2}}
$$

and values for the three samples are included in Table 4. It is clear that the $K^{*}$ values for the three samples are quite similar, varying by at most $5 \%$ from the average value, thereby demonstrating that for samples with similar porosity such as those considered here, the permeability $K$ is a strong function of pore diameter. Samples with a larger pore diameter exhibit a large permeability and vice-versa. Similar observations have been made by [30].

\subsubsection{Metal matrix features}

Features of the metal matrix such as ligament cross-section and ligament length govern the heat conduction through the foam sample. The distributions of ligament length and corrected ligament area of cross-section, after inclusion of parameter $\Delta A$ (Eq.(9)) are shown in Fig. 9 and Fig. 10, respectively. The distributions indicate a fair amount of randomness in the foam samples. Though clear peaks close to the average values are observed, standard deviations are of the order of the average values themselves, for both the ligament area of cross-section and ligament length. Table 5 shows the ligament length and ligament area of cross-section non-dimensionalized as: 
As with the pore diameters, the probability densities of ligament length and effective ligament area of cross-section are fitted using Fourier polynomials of order 8 and 10, respectively, as follows:

$$
\begin{aligned}
& p\left(l_{\text {lig }}\right)=k+\sum_{i=1}^{8} a_{i} \cos \left(i l_{\text {lig }}\right)+\sum_{i=1}^{8} a_{i} \sin \left(i l_{\text {lig }}\right) \\
& p\left(A_{\text {lig }}\right)=k+\sum_{i=1}^{10} a_{i} \cos \left(i A_{\text {lig }}\right)+\sum_{i=1}^{10} a_{i} \sin \left(i A_{\text {lig }}\right)
\end{aligned}
$$

The corresponding coefficients for Eqs. (16) and (17) are provided in Table 6 and Table 7, respectively, along with the range of applicability for each foam sample.

The distributions of co-ordination number and node density are shown respectively in Fig. 11 and Fig. 12. Co-ordination number is the number of neighboring nodes surrounding each node, while node density is the number of nodes per $\mathrm{cm}^{3}$ volume of the sample. The results of nodes per unit volume are computed by discretizing the foam sample into cubic volumes, with sides of $2.5 \mathrm{~mm}, 2.5 \mathrm{~mm}$ and $1.6 \mathrm{~mm}$ for the $10 \mathrm{ppi}, 20 \mathrm{ppi}$ and 40 ppi samples, respectively. More than two-thirds of the total number of nodes in each case demonstrate a co-ordination number of 3 or 4 . Again, all the three foam samples show a similar distribution, further corroborating the similarity in their structure. The probability density function of the node density is fitted as a ratio of two polynomial expressions given below:

$$
p(x)=\frac{a+c x+e x^{2}+g x^{3}+i x^{4}}{1+b x+d x^{2}+f x^{3}+h x^{4}+j x^{5}}
$$


Here $x$ is the node density, and the values of the coefficients in Eq. (18) are listed in Table 8.

\section{Conclusions}

Imaging-based analytical modeling of realistic porous media is performed in this work. Employing an analogy between electrical and thermal conduction, a new resistance network model for effective thermal conductivity is presented. It is demonstrated that approximate yet very fast computations of effective thermal conductivity may be performed with the proposed model. Further, through microstructural characterization of metal and pore features, it is observed that all the three foam samples are structurally similar and the microstructural features scale with the pore size of the samples. It is further demonstrated that real foam samples exhibit a distribution of pore features and that the pores are not exactly spherical. For samples with similar porosity, a strong dependence of permeability on pore size is also demonstrated employing previously computed permeability data using detailed CFD analysis on the same samples.

\section{Acknowledgments}

The authors acknowledge the assistance of Dr. Eric Nauman of Purdue University for kindly scanning the foam samples. Support for this work from the Cooling Technologies Research Center, an NSF Industry/University Cooperative Research Center at Purdue University, is also gratefully acknowledged. 


\section{References}

[1] M.F. Ashby, A. Evans, N.A. Fleck, L.J. Gibson, J.W. Hutchinson, H.J.G. Wadley, Metal foams: A Design Guide, Boston, Butterworth-Heinemann, 2000.

[2] B. Ozmat, B. Leyda, B. Benson, Thermal Applications of Open-Cell Metal Foams, Materials and Manufacturing Processes. 19 (2004) 839 - 862.

[3] T.J. Lu, H.A. Stone, M.F. Ashby, Heat Transfer in Open-Cell Metal Foams, Acta Materialia. 46 (1998) 3619-3635.

[4] K. Boomsma, D. Poulikakos, F. Zwick, Metal Foams as Compact High Performance Heat Exchangers, Mechanics of Materials. 35 (2003) 1161-1176.

[5] V. Calmidi, R. Mahajan, Effective Thermal Conductivity of High Porosity Fibrous Metal Foams, Journal of Heat Transfer. 121 (1999) 466-471.

[6] J. Paek, B. Kang, S. Kim, J. Hyun, Effective Thermal Conductivity and Permeability of Aluminum Foam Materials, International Journal of Thermophysics. 21 (2000) 453-464.

[7] K. Boomsma, D. Poulikakos, On the Effective Thermal Conductivity of a Three-Dimensionally Structured Fluid-Saturated Metal Foam, International Journal of Heat and Mass Transfer. 44 (2001) 827-36.

[8] S. Krishnan, J.Y. Murthy, S.V. Garimella, Direct Simulation of Transport in Open-Cell Metal Foam, J. Heat Transfer. 128 (2006) 793-799.

[9] P. Du Plessis, A. Montillet, J. Comiti, J. Legrand, Pressure Drop Prediction for Flow through High Porosity Metallic Foams, Chemical Engineering Science. 49 (1994) 3545-3553.

[10] T. Fiedler, I. Belova, A. Ochsner, G. Murch, Non-Linear Calculations of Transient Thermal Conduction in Composite Materials, Computational Materials Science. 45 (2009) 434-438.

[11] G. Laschet, J. Sauerhering, O. Reutter, T. Fend, J. Scheele, Effective Permeability and Thermal Conductivity of Open-Cell Metallic Foams via Homogenization on a Microstructure Model, Computational Materials Science. 45 (2009) 597-603.

[12] K.K. Bodla, J.Y. Murthy, S.V. Garimella, XMT-Based Direct Simulation of Flow and Heat Transfer through Open-cell Aluminum Foams, in: 2010 13th Intersociety Conference on Thermal and Thermomechanical Phenomena in Electronic Systems (ITHERM '10), June 2-June 5, Las Vegas, NV, USA, IEEE, 2010.

[13] C. Redenbach, Microstructure Models for Cellular Materials, Computational Materials Science. 44 (2009) 1397-1407.

[14] J. Staggs, Convection Heat Transfer in the Cone Calorimeter, Fire Safety Journal. 44 (2009) 469474.

[15] I. Fatt, Network Model of Porous Media, Journal of Petroleum Technology. 8 (1956) 144-177.

[16] A. Raoof, S. Majid Hassanizadeh, A New Method for Generating Pore-Network Models of Porous Media, Transport in Porous Media. 81 (2010) 391-407.

[17] S.R. Annapragada, J.Y. Murthy, S.V. Garimella, Permeability and Thermal Transport in Compressed Open-Celled Foams, Numerical Heat Transfer, Part B: Fundamentals. 54 (2008) 1-22.

[18] J. Banhart, Manufacture, Characterisation and Application of Cellular Metals and Metal Foams, Progress in Materials Science. 46 (2001) 559-632.

[19] DUOCEL Aluminum Foam Properties, ERG Materials and Aerospace Corporation.

[20] AMIRA online user's guide for AMIRA 5, 2009.

[21] H. Blum, A Transformation for Extracting New Descriptors of Shape, in: Models for the Perception of Speech and Visual Form, Cambridge, MA, MIT press, 1967: pp. 362-380.

[22] M. Paradowski, H. Kwasnicka, K. Borysewicz, Capillary Blood Vessel Tortuosity Measurement using Graph Analysis, in: 13th International Conference on Knowledge-Based and Intelligent Information and Engineering Systems (KES '09), September 28 - September 30, Santiago, Chile, Springer Verlag, 2009: pp. 135-142.

[23] K. Palagyi, G. Nemeth, Fully Parallel 3D Thinning Algorithms Based on Sufficient Conditions for 
Topology Preservation, in: Discrete Geometry for Computer Imagery: 15th IAPR International Conference (DGCI '09), September 30-October 2, Montréal, Canada, Springer-Verlag New York Inc, 2009: pp. 481-492.

[24] F.P. Incropera, D.P. DeWitt, Fundamentals of Heat and Mass Transfer, New York: John Wiley \& Sons, 1990.

[25] S. Krishnan, S.V. Garimella, J.Y. Murthy, Simulation of Thermal Transport in Open-Cell Metal Foams: Effect of Periodic Unit-Cell Structure, Journal of Heat Transfer. 130 (2008) 1-5.

[26] AVIZO user's guide for AVIZO 6.2, 2009.

[27] R. Lemlich, A Theory for the Limiting Conductivity of Polyhedral Foam at Low Density, Journal of Colloid and Interface Science. 64 (1978) 107-110.

[28] A. Bhattacharya, V. Calmidi, R. Mahajan, Thermophysical Properties of High Porosity Metal Foams, International Journal of Heat and Mass Transfer. 45 (2002) 1017-1031.

[29] F. Meyer, Topographic distance and watershed lines, Signal Process. 38 (1994) 113-125.

[30] M. Medraj, E. Baril, V. Loya, L. Lefebvre, The Effect of Microstructure on the Permeability of Metallic Foams, Journal of Materials Science. 42 (2007) 4372-4383. 


\section{LIST OF FIGURE CAPTIONS}

Fig. 1. Image processing steps: a) Original image, b) cropped region of interest after filtering, and c) resampled image.

Fig. 2. Image segmentation: a) greyscale image, b) segmented (binarised) image, and c) greyscale image histogram.

Fig. 3. a) A 10 ppi foam sample, b) its corresponding image skeleton, and c) resistance network surrounding one sample node.

Fig. 4. Temperature contours predicted for conduction along the longest dimension in a) $10 \mathrm{ppi}$, b) $20 \mathrm{ppi}$, and c) 40 ppi foam sample (also shown are the boundary zones and actual conducting length).

Fig. 5. Temperature contours in 10 ppi foam sample, reproduced from [12].

Fig. 6. Effective thermal conductivity as a function of porosity.

Fig. 7. Pores identified by color in 10 ppi foam sample.

Fig. 8. Distribution of effective pore diameters. Mean and standard deviation values may be found in Table 2.

Fig. 9. Distribution of ligament lengths.

Fig. 10. Distribution of ligament areas of cross-section.

Fig. 11. Distribution of node co-ordination numbers.

Fig. 12. Distribution of node density. 


\section{LIST OF TABLE CAPTIONS}

Table 1. Average effective thermal conductivity predictions.

Table 2. Pore diameter measurements.

Table 3. Parameters for Probability Density Function (PDF) of effective pore diameter.

Table 4. Sphericity and normalized permeability predictions.

Table 5. Normalized ligament length and ligament area of cross-section measurements.

Table 6. Parameters for PDF of ligament length.

Table 7. Parameters for PDF of ligament area of cross-section.

Table 8. Parameters for PDF of node density. 

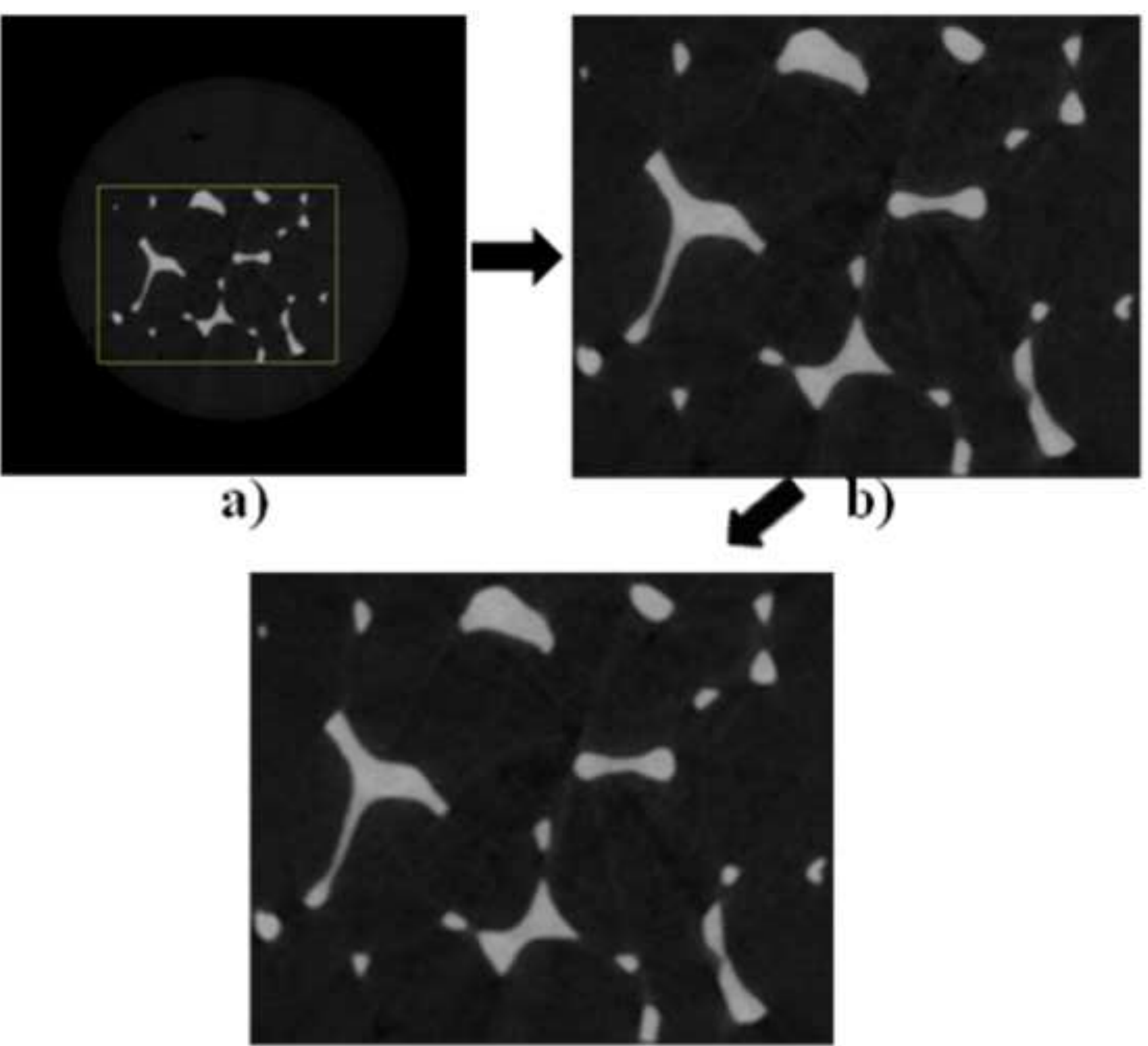

c) 
Click here to download high resolution image

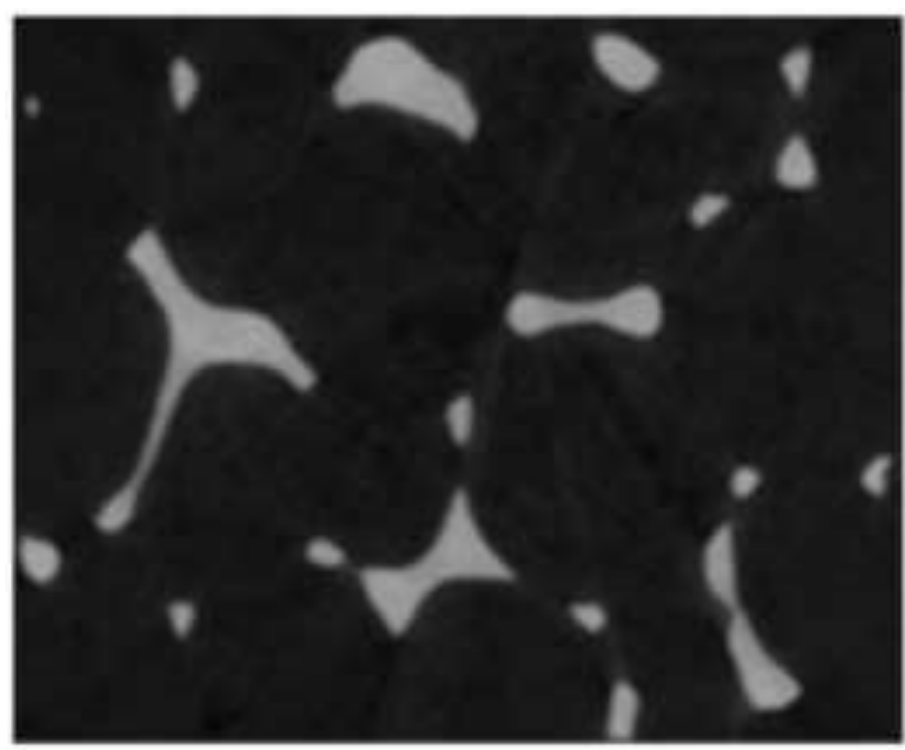

a)

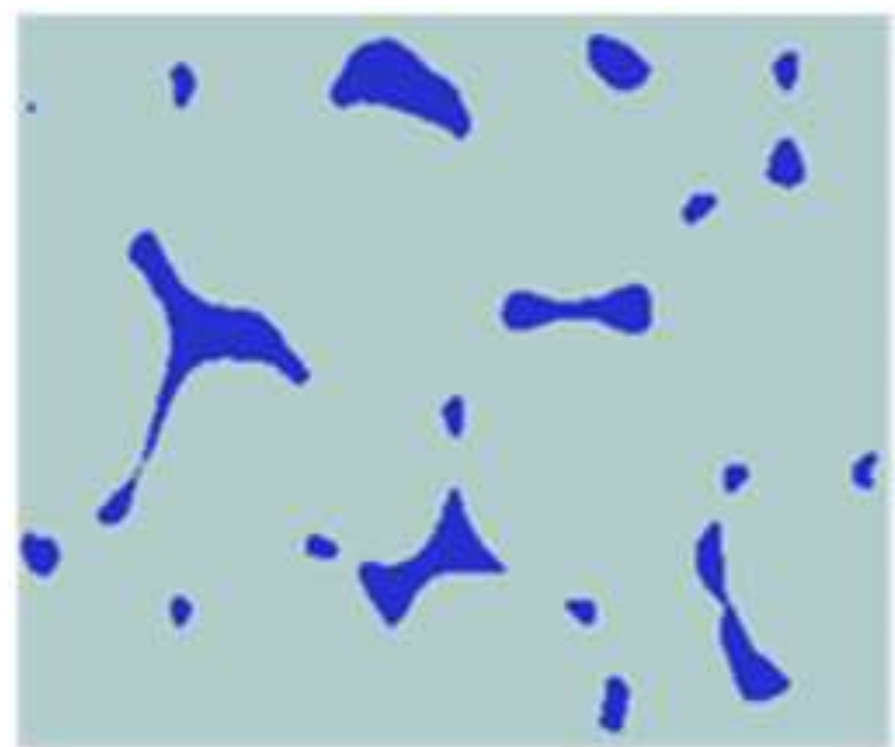

b)

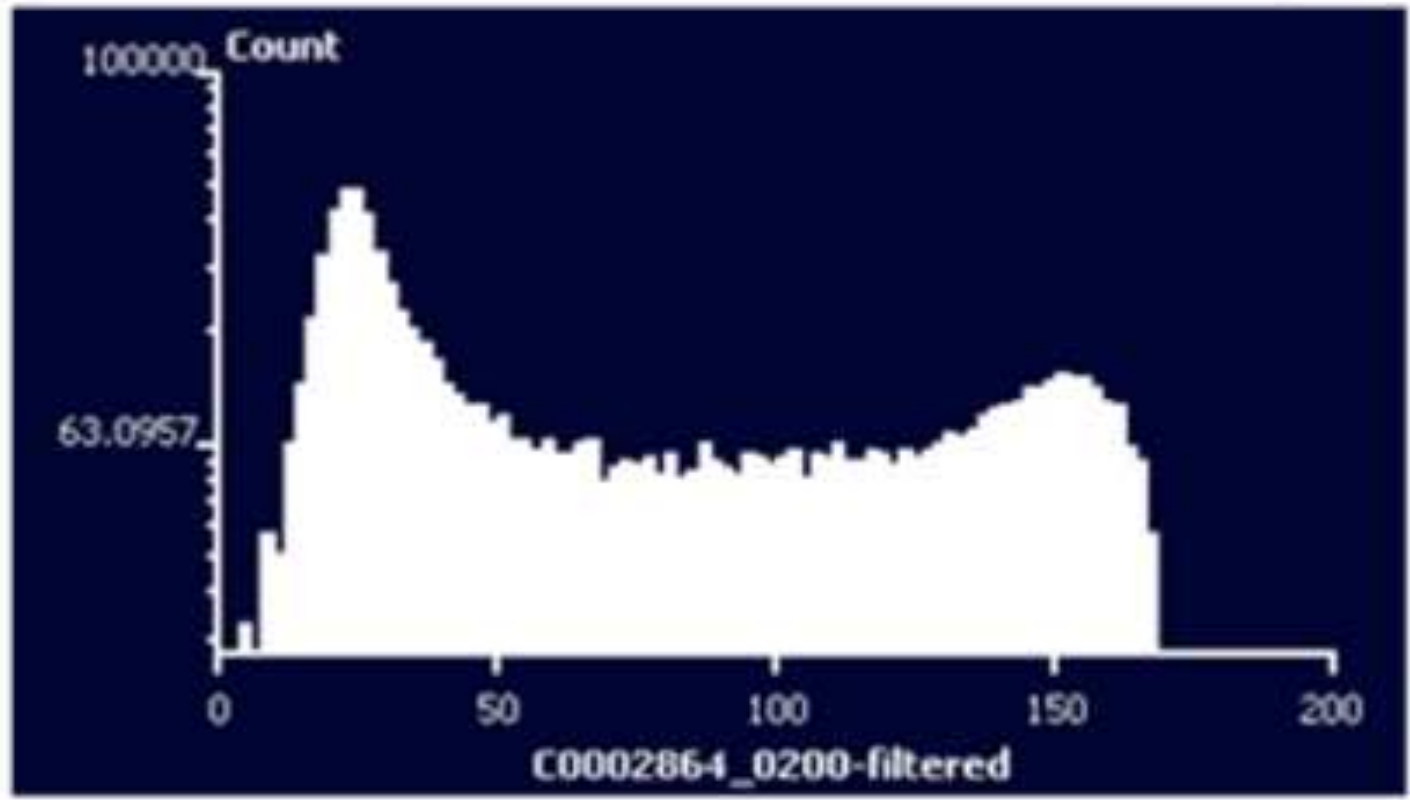

c) 
Click here to download high resolution image

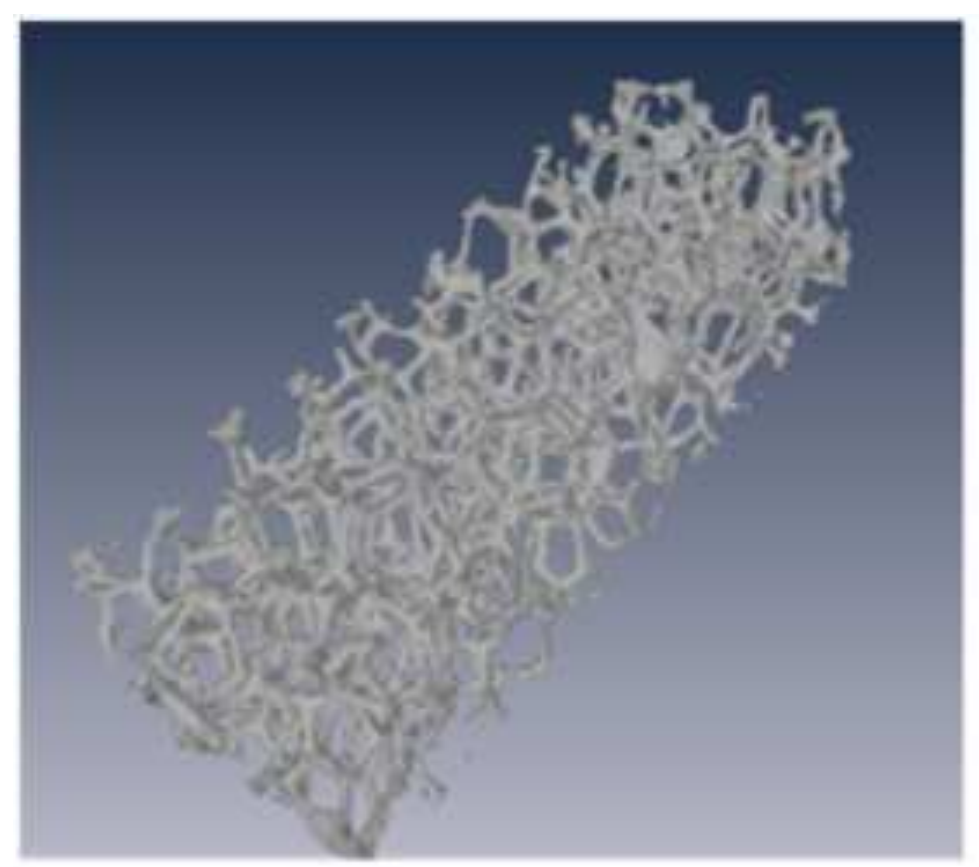

a)

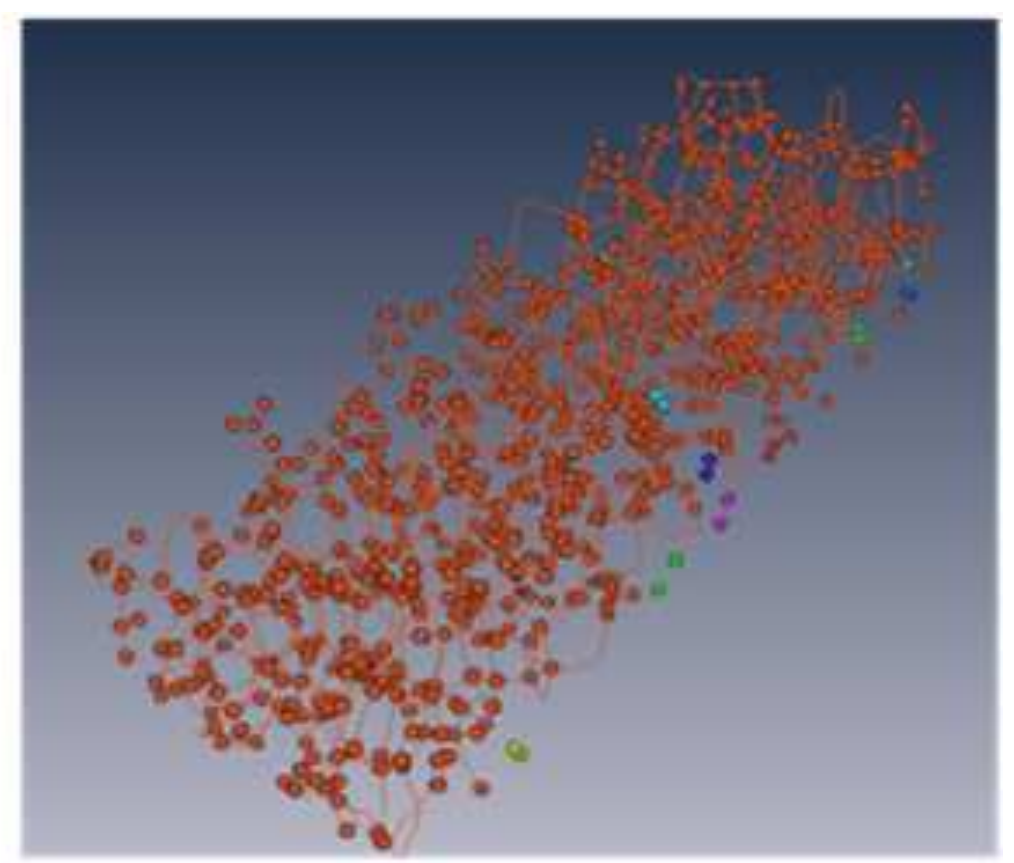

b)

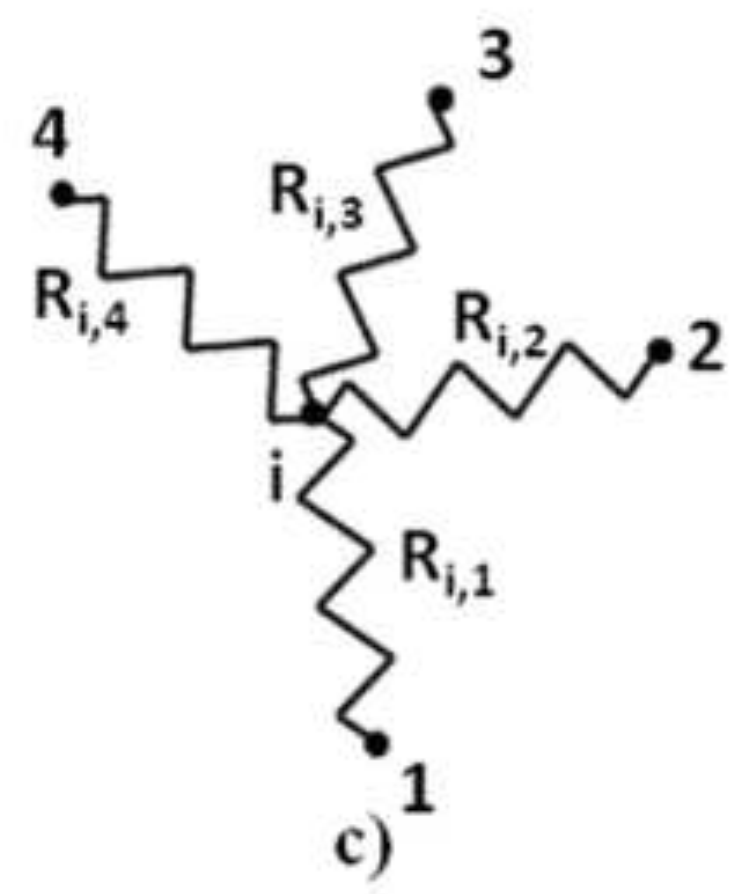




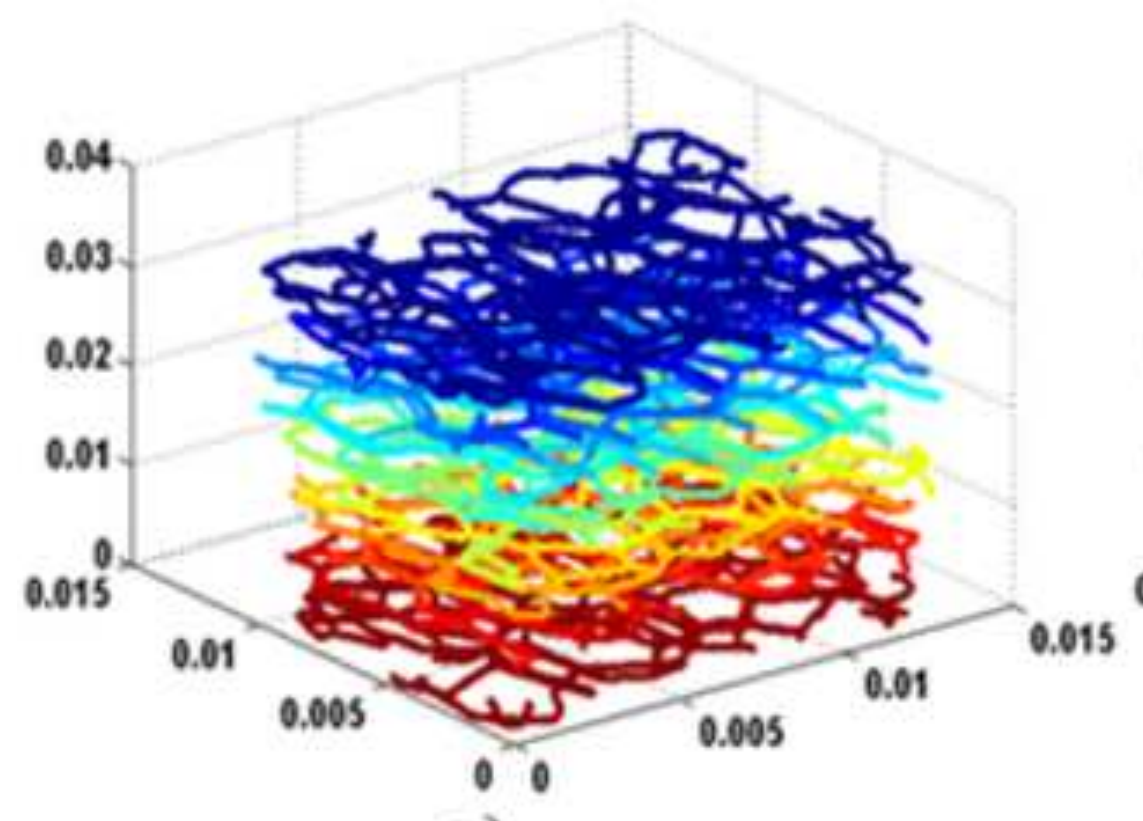

a)

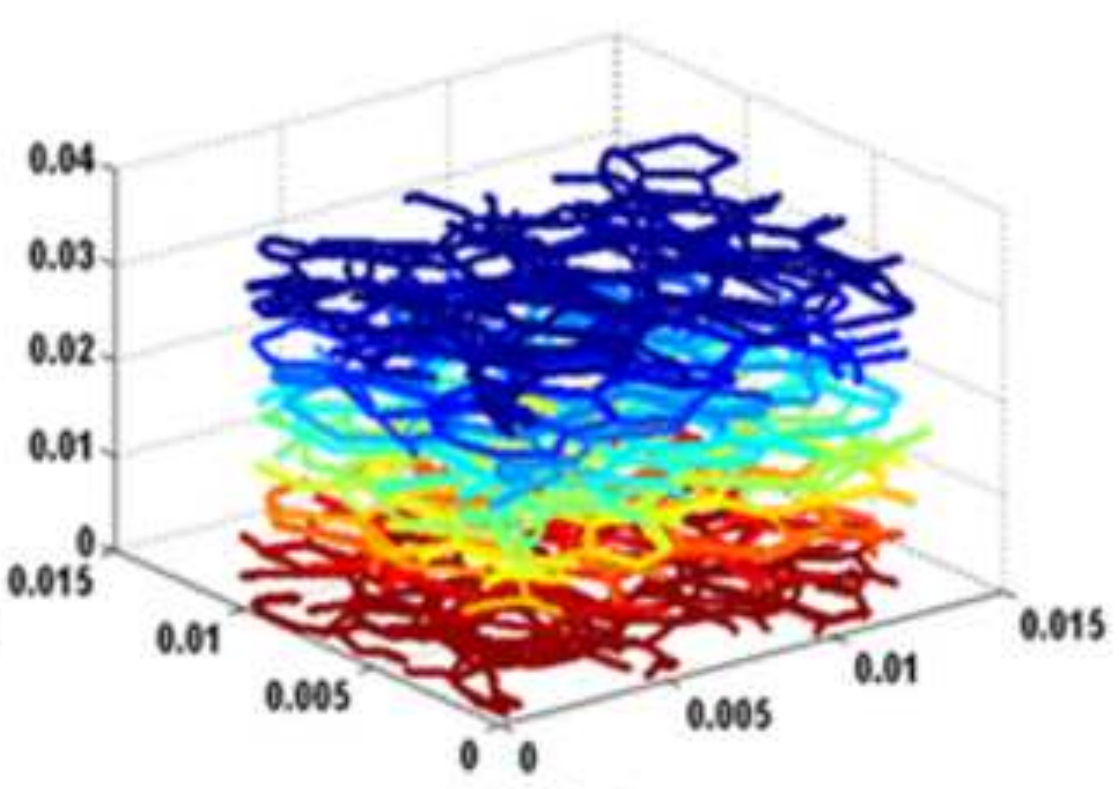

b)

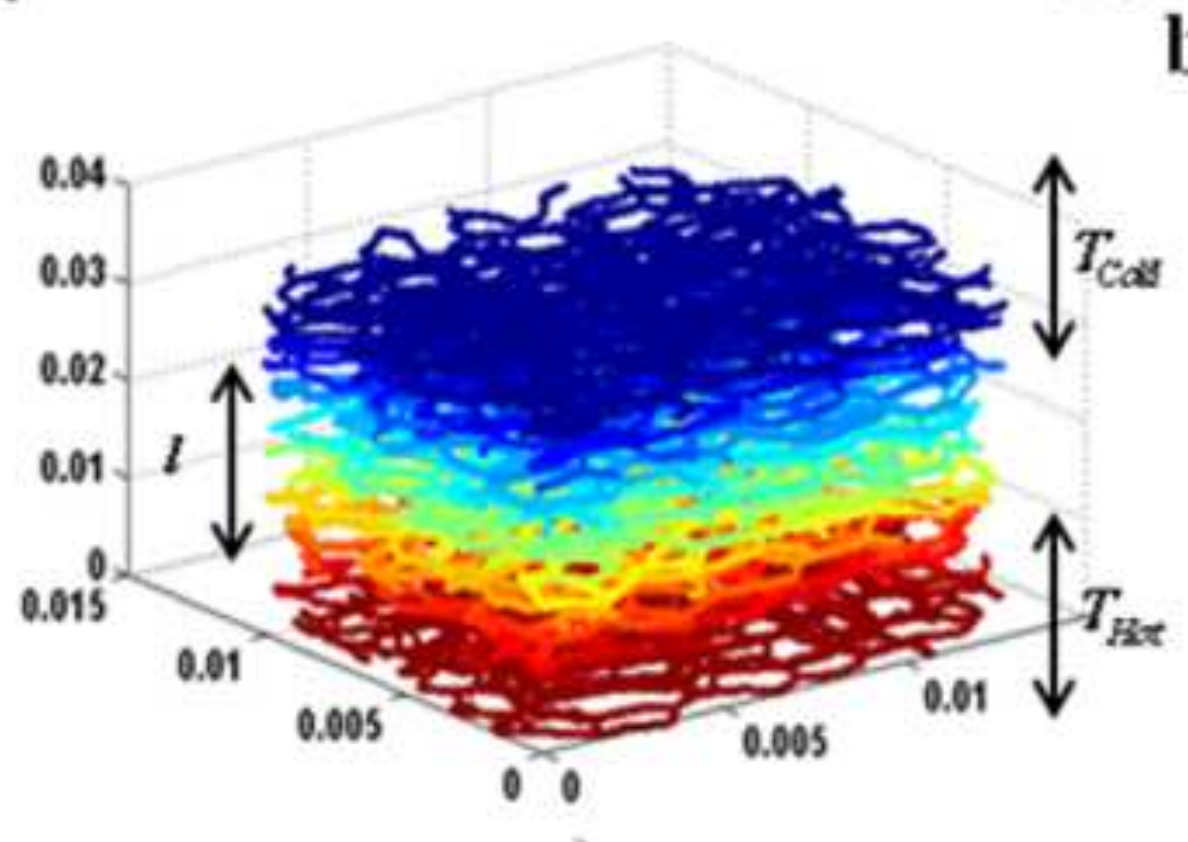

c)

40
30
10
0


Click here to download high resolution image

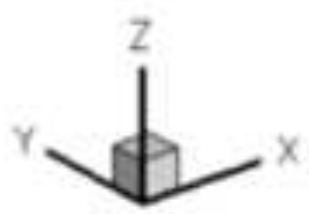

Temperalure
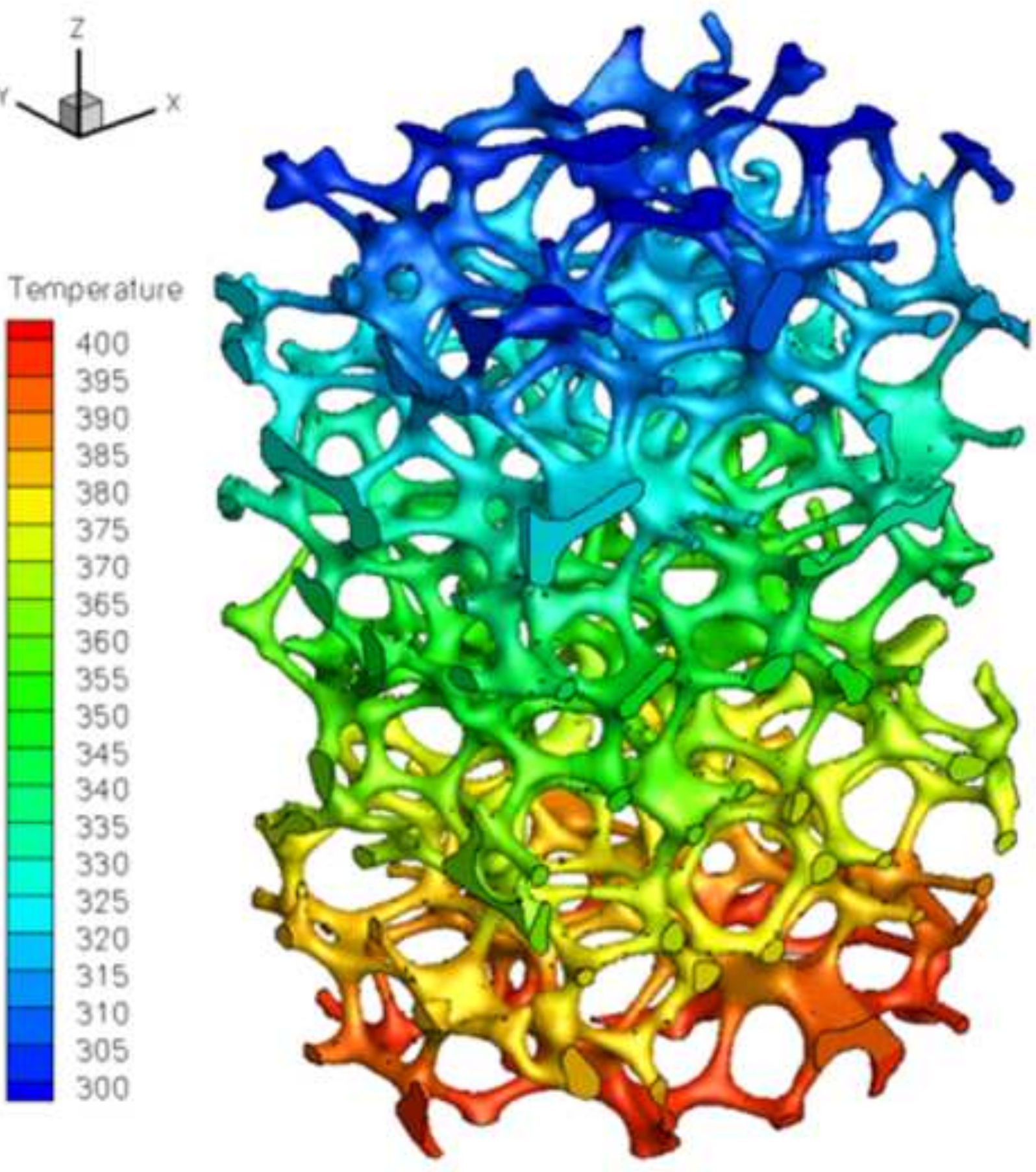


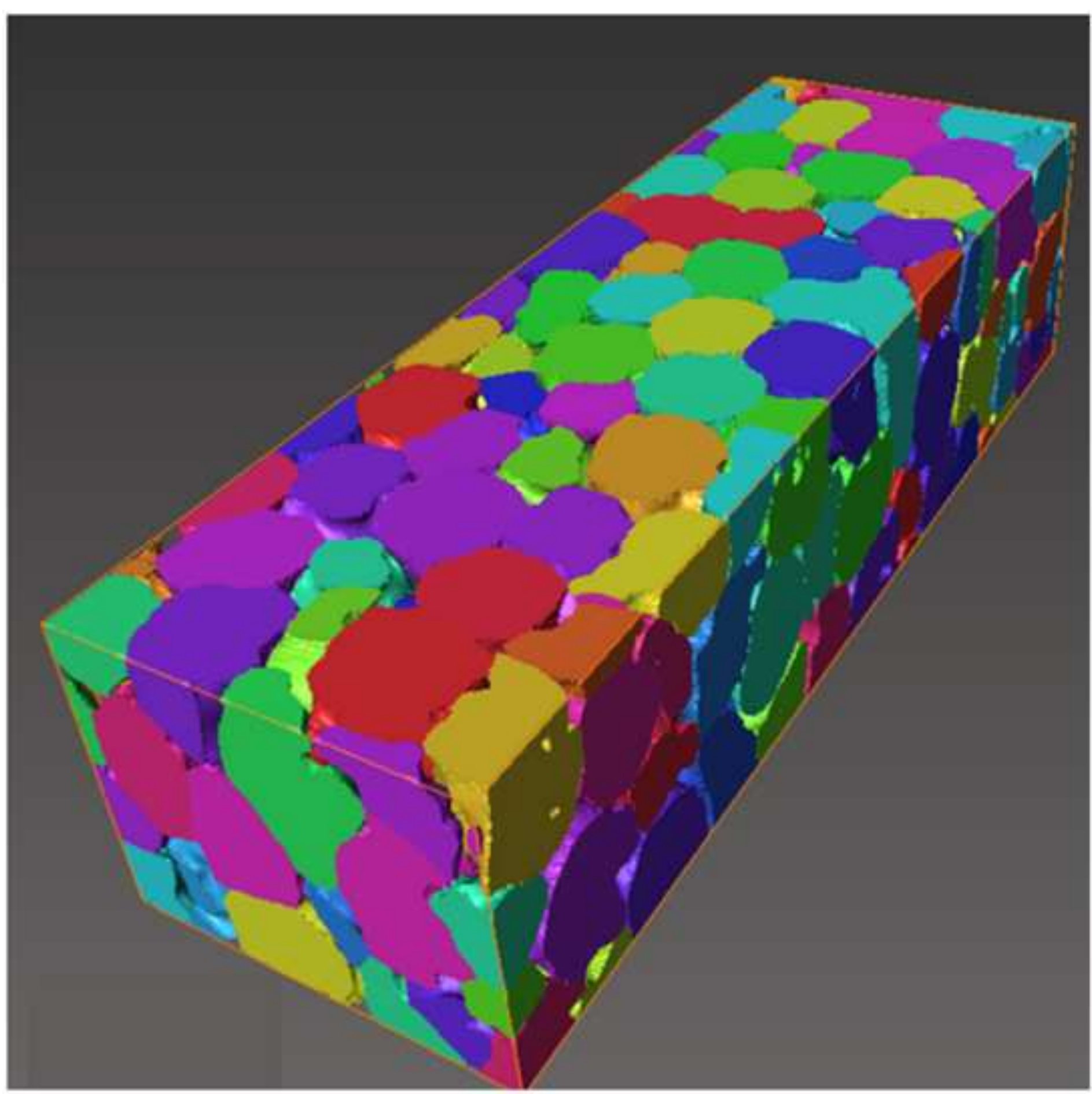




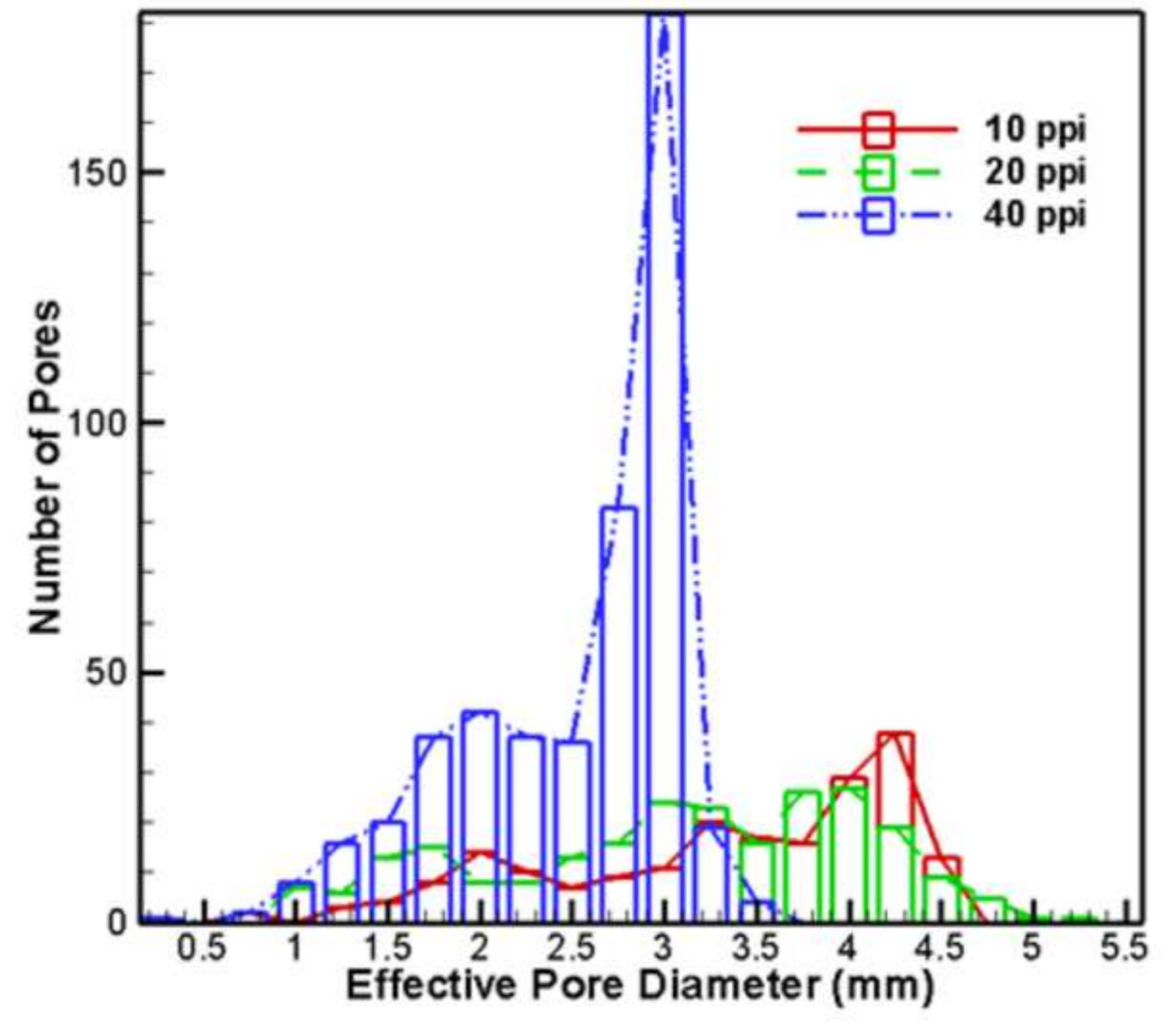

Figure 8
Click here to download high resolution image 


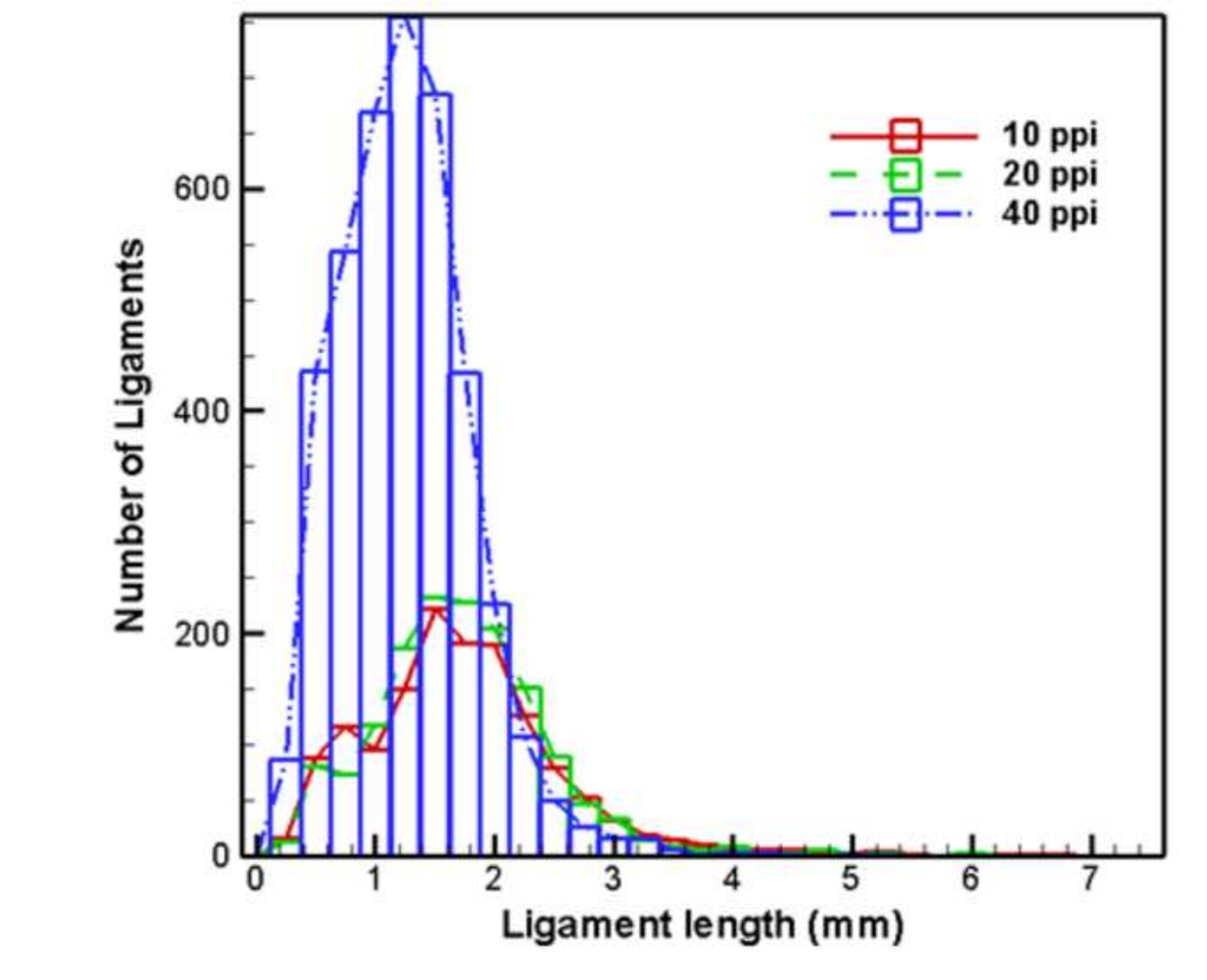

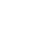


Click here to download high resolution image

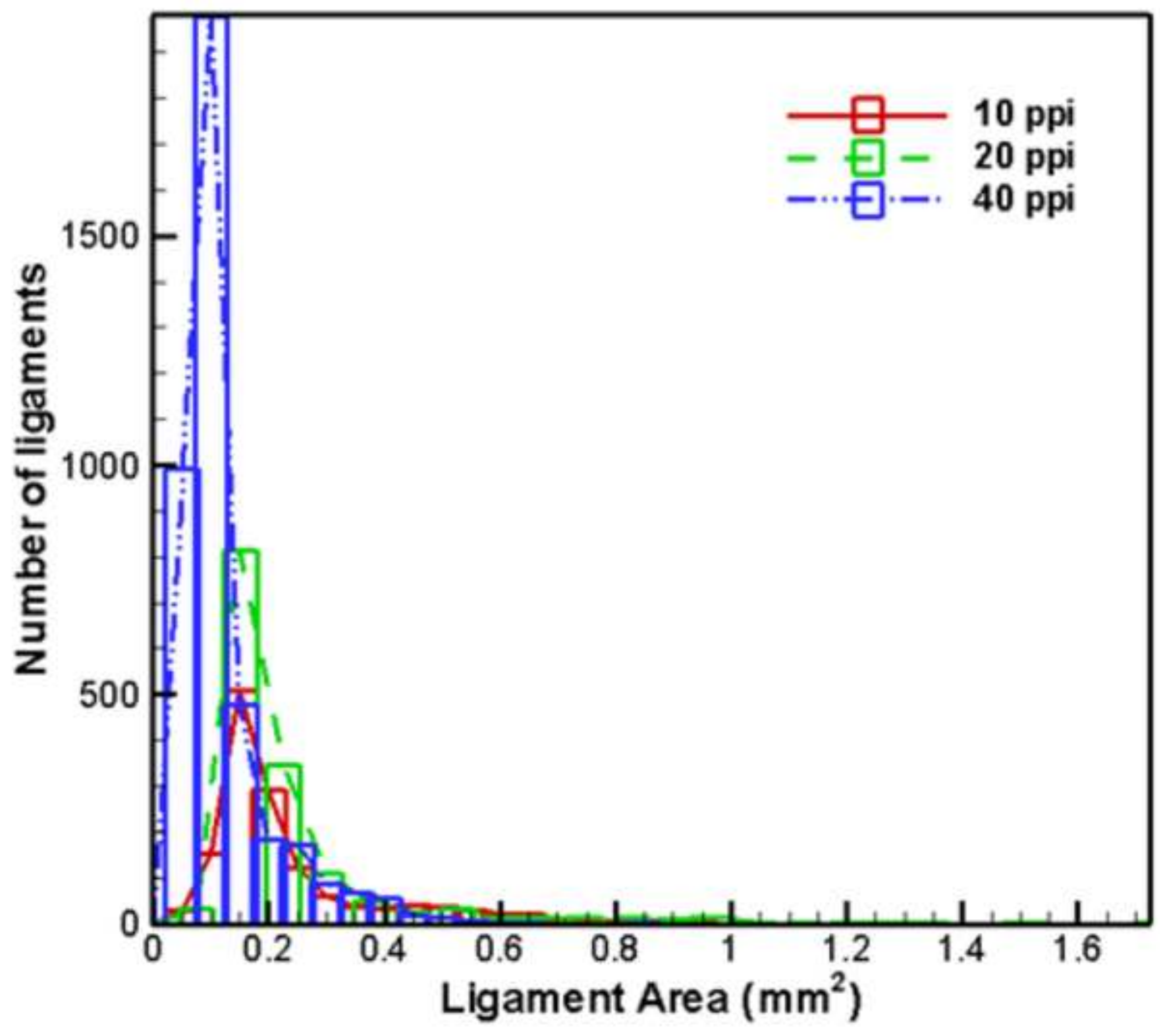




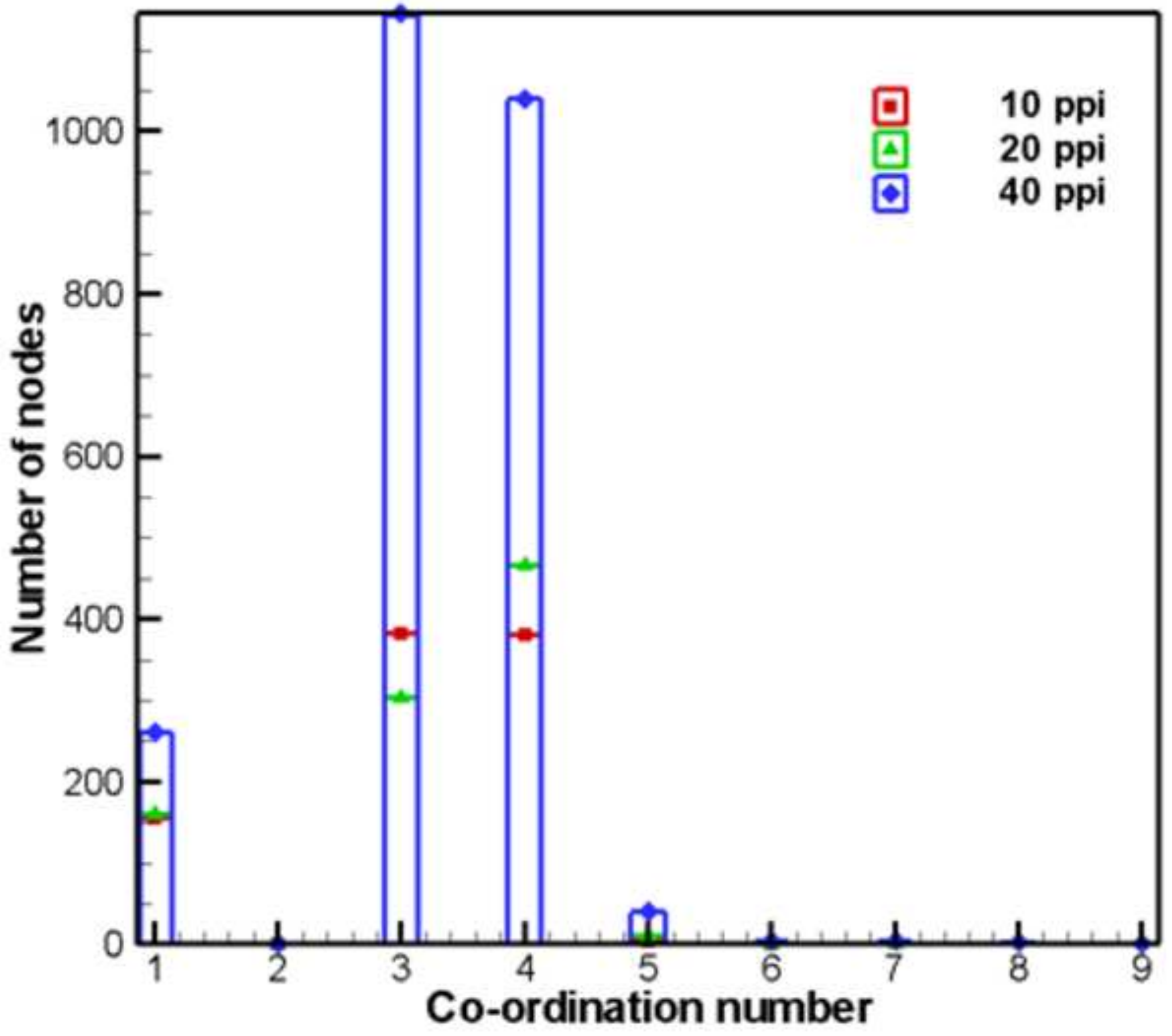




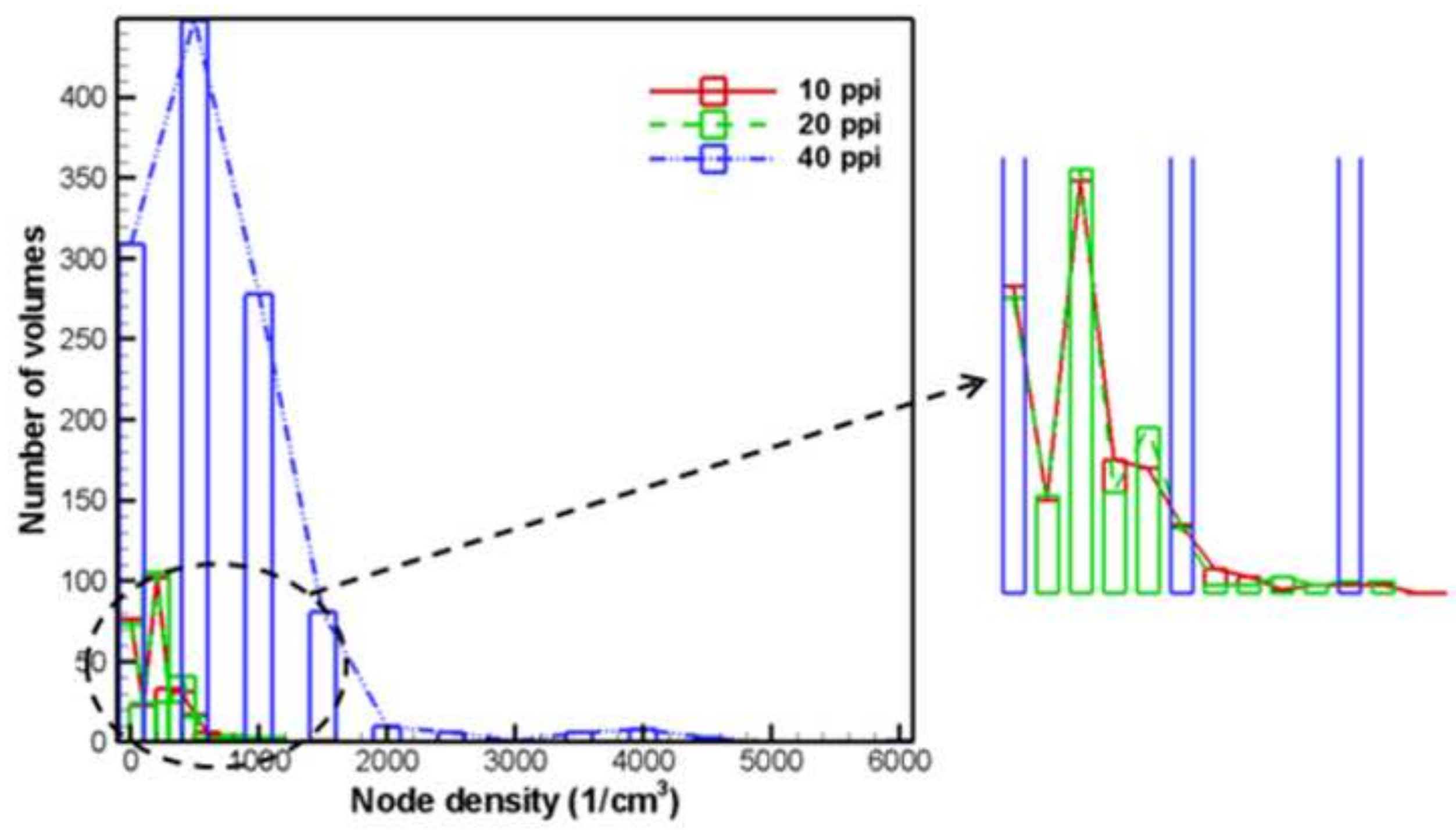




\begin{tabular}{cccc}
\hline Sample & $\boldsymbol{\varepsilon}_{\boldsymbol{v}}(\%)$ & $\begin{array}{c}\boldsymbol{k}_{\text {eff }}(\mathbf{W} / \mathbf{m} . \mathbf{k}) \\
(\text { Present })\end{array}$ & $\begin{array}{c}\boldsymbol{k}_{\text {eff,air }}(\mathbf{W} / \mathbf{m} . \mathbf{k}) \\
(\mathbf{X M T}+\mathbf{C F D})\end{array}$ \\
\hline $10 \mathrm{ppi}$ & 91.31 & 5.20 & 5.34 \\
$20 \mathrm{ppi}$ & 91.26 & 5.21 & 6.59 \\
$40 \mathrm{ppi}$ & 91.98 & 4.52 & 5.35 \\
\hline
\end{tabular}




\begin{tabular}{cccc}
\hline Sample & Inverse ppi $(\mathbf{m m})$ & $\begin{array}{c}\text { Pore diameter, } \boldsymbol{d}_{\text {pore }} \\
(\mathbf{m m})\end{array}$ & $\begin{array}{c}\text { Standard deviation } \\
\text { in } \boldsymbol{d}_{\text {pore }}(\mathbf{m m})\end{array}$ \\
\hline $10 \mathrm{ppi}$ & 2.540 & 3.214 & 0.929 \\
$20 \mathrm{ppi}$ & 1.270 & 2.976 & 0.999 \\
$40 \mathrm{ppi}$ & 0.635 & 2.406 & 0.593 \\
\hline
\end{tabular}




\begin{tabular}{|c|c|c|c|c|c|c|}
\hline Co-efficients & 10 ppi & $\begin{array}{c}\text { Range } \\
(\mathrm{mm})\end{array}$ & 20 ppi & $\begin{array}{c}\text { Range } \\
(\mathrm{mm})\end{array}$ & 40 ppi & $\begin{array}{c}\text { Range } \\
(\mathbf{m m})\end{array}$ \\
\hline$k$ & 68.16 & & 85.76 & & -11270.30 & \\
\hline$a_{1}$ & 3.76 & & -0.76 & & -121.70 & \\
\hline$a_{2}$ & -105.57 & & -132.60 & & 17396.27 & \\
\hline$a_{3}$ & -6.73 & & -1.44 & & 207.73 & \\
\hline$a_{4}$ & 47.89 & & 59.81 & & -7801.82 & \\
\hline$a_{5}$ & 3.78 & & -0.92 & & -104.04 & \\
\hline$a_{6}$ & -11.70 & & -14.45 & & 1860.56 & \\
\hline$a_{7}$ & -0.87 & & 0.26 & & 18.48 & \\
\hline$a_{8}$ & 1.24 & & 1.51 & & -188.47 & \\
\hline$a_{9}$ & 0.06 & & -0.03 & & -0.48 & \\
\hline$a_{10}$ & -0.03 & $0.0-5.0$ & -0.03 & $0.0-6.0$ & 3.71 & $0.2-4.0$ \\
\hline$b_{1}$ & -127.89 & & -160.85 & & 21134.67 & \\
\hline$b_{2}$ & -6.22 & & 1.28 & & 197.49 & \\
\hline$b_{3}$ & 76.29 & & 95.58 & & -12513.50 & \\
\hline$b_{4}$ & 5.63 & & -1.26 & & -165.87 & \\
\hline$b_{5}$ & -25.80 & & -32.07 & & 4161.22 & \\
\hline$b_{6}$ & -2.04 & & 0.54 & & 50.84 & \\
\hline$b_{7}$ & 4.33 & & 5.31 & & -675.33 & \\
\hline$b_{8}$ & 0.028 & & -0.10 & & -4.47 & \\
\hline$b_{9}$ & -0.25 & & -0.30 & & 36.42 & \\
\hline$b_{10}$ & -0.01 & & 0.01 & & -0.05 & \\
\hline
\end{tabular}




\begin{tabular}{ccccc}
\hline Sample & Sphericity, $\boldsymbol{\Psi}$ & $\begin{array}{c}\text { Pore diameter, } \\
\boldsymbol{d}_{\text {pore }}(\mathbf{m m})\end{array}$ & $\begin{array}{c}\text { Permeability, } \boldsymbol{K} \\
\left(\mathbf{m m}^{2}\right)\end{array}$ & $\begin{array}{c}\text { Normalized } \\
\text { Permeability, } \boldsymbol{K}^{*}\end{array}$ \\
\hline $10 \mathrm{ppi}$ & 0.766 & 3.214 & $1.72 \times 10^{-07}$ & $1.68 \times 10^{-02}$ \\
$20 \mathrm{ppi}$ & 0.751 & 2.976 & $1.60 \times 10^{-07}$ & $1.81 \times 10^{-02}$ \\
$40 \mathrm{ppi}$ & 0.791 & 2.406 & $1.02 \times 10^{-07}$ & $1.75 \times 10^{-02}$ \\
\hline
\end{tabular}




\begin{tabular}{ccc}
\hline Sample & Ligament length $\left(\boldsymbol{l}^{*}\right)$ & $\begin{array}{c}\text { Ligament } \\
\text { Area }\left(\boldsymbol{A}_{\text {lig }}{ }^{*}\right)\end{array}$ \\
\hline $10 \mathrm{ppi}$ & 0.498 & $1.83 \times 10^{-02}$ \\
$20 \mathrm{ppi}$ & 0.537 & $2.04 \times 10^{-02}$ \\
$40 \mathrm{ppi}$ & 0.469 & $1.50 \times 10^{-02}$ \\
\hline
\end{tabular}




\begin{tabular}{|c|c|c|c|c|c|c|}
\hline Co-efficients & 10 ppi & $\begin{array}{c}\text { Range } \\
(\mathrm{mm})\end{array}$ & 20 ppi & $\begin{array}{c}\text { Range } \\
(\mathbf{m m})\end{array}$ & 40 ppi & $\begin{array}{c}\text { Range } \\
\text { (mm) }\end{array}$ \\
\hline$k$ & 34.61 & & 118.35 & & 141.96 & \\
\hline$a_{1}$ & 12.12 & & 6.34 & & 20.51 & \\
\hline$a_{2}$ & -47.54 & & -171.09 & & -203.14 & \\
\hline$a_{3}$ & -18.95 & & -10.02 & & -32.48 & \\
\hline$a_{4}$ & 14.07 & & 61.43 & & 70.40 & \\
\hline$a_{5}$ & 7.63 & & 4.15 & & 13.52 & \\
\hline$a_{6}$ & -1.12 & & -8.93 & & -9.42 & \\
\hline$a_{7}$ & -0.80 & & -0.47 & & -1.55 & \\
\hline$a_{8}$ & -0.02 & $0.0-6.5$ & 0.25 & $0.0-6.0$ & 0.20 & $0.0-6.5$ \\
\hline$b_{1}$ & -63.06 & & -218.37 & & -261.30 & \\
\hline$b_{2}$ & -19.02 & & -9.97 & & -32.36 & \\
\hline$b_{3}$ & 29.18 & & 112.77 & & 132.07 & \\
\hline$b_{4}$ & 13.88 & & 7.42 & & 24.10 & \\
\hline$b_{5}$ & -5.01 & & -26.86 & & -29.78 & \\
\hline$b_{6}$ & -3.05 & & -1.71 & & -5.59 & \\
\hline$b_{7}$ & 0.07 & & 2.04 & & 1.97 & \\
\hline$b_{8}$ & 0.10 & & 0.07 & & 0.22 & \\
\hline
\end{tabular}




\begin{tabular}{|c|c|c|c|c|c|c|}
\hline Co-efficients & 10 ppi & $\begin{array}{c}\text { Range } \\
\left(\mathbf{m m}^{2}\right)\end{array}$ & 20 ppi & $\begin{array}{l}\text { Range } \\
\left(\mathbf{m m}^{2}\right)\end{array}$ & 40 ppi & $\begin{array}{l}\text { Range } \\
\left(\mathbf{m m}^{2}\right)\end{array}$ \\
\hline$k$ & 15964.46 & & 12724.85 & & 11884.01 & \\
\hline$a_{1}$ & 513.59 & & 2722.49 & & 1611.74 & \\
\hline$a_{2}$ & -24517.70 & & -18969.30 & & -17916.10 & \\
\hline$a_{3}$ & -931.79 & & -4721.80 & & -2795.52 & \\
\hline$a_{4}$ & 10814.66 & & 7597.69 & & 7454.42 & \\
\hline$a_{5}$ & 538.79 & & 2479.84 & & 1468.01 & \\
\hline$a_{6}$ & -2494.15 & & -1448.62 & & -1544.24 & \\
\hline$a_{7}$ & -130.24 & & -510.10 & & -301.69 & \\
\hline$a_{8}$ & 236.83 & & 96.11 & & 123.52 & \\
\hline$a_{9}$ & 9.65 & & 29.57 & & 17.46 & \\
\hline$a_{10}$ & -4.08 & $0.01-1.0$ & -0.72 & $0.05-2.0$ & -1.66 & $0.02-1.0$ \\
\hline$b_{1}$ & -29900.20 & & -23658.40 & & -22155.90 & \\
\hline$b_{2}$ & -852.19 & & -4442.87 & & -2630.29 & \\
\hline$b_{3}$ & 17519.37 & & 13038.49 & & 12500.89 & \\
\hline$b_{4}$ & 789.14 & & 3837.97 & & 2272.25 & \\
\hline$b_{5}$ & -5687.66 & & -3686.75 & & -3741.53 & \\
\hline$b_{6}$ & -297.52 & & -1275.18 & & -754.64 & \\
\hline$b_{7}$ & 881.31 & & 440.69 & & 505.40 & \\
\hline$b_{8}$ & 43.03 & & 150.76 & & 89.08 & \\
\hline$b_{9}$ & -43.32 & & -12.91 & & -20.14 & \\
\hline$b_{10}$ & -1.11 & & -2.92 & & -1.72 & \\
\hline
\end{tabular}




\begin{tabular}{|c|c|c|c|c|c|c|}
\hline Co-efficients & 10 ppi & $\begin{array}{r}\text { Range } \\
\left(\mathrm{cm}^{-3}\right)\end{array}$ & 20 ppi & $\begin{array}{c}\text { Range } \\
\left(\mathrm{cm}^{-3}\right)\end{array}$ & 40 ppi & $\begin{array}{l}\text { Range } \\
\left(\mathrm{cm}^{-3}\right)\end{array}$ \\
\hline$a$ & 0.11 & & 0.08 & & 0.23 & \\
\hline$b$ & -0.01 & & -0.01 & & 0.00 & \\
\hline$c$ & 0.00 & & 0.00 & & 0.00 & \\
\hline$d$ & $2.88 \times 10^{-05}$ & & $2.90 \times 10^{-05}$ & & $-3.90 \times 10^{-06}$ & \\
\hline$e$ & $8.22 \times 10^{-07}$ & & $8.36 \times 10^{-07}$ & & $1.41 \times 10^{-07}$ & \\
\hline$f$ & $-5.40 \times 10^{08}$ & $0-1450$ & $-5.30 \times 10^{-08}$ & $0-1100$ & $3.78 \times 10^{-09}$ & $0-5600$ \\
\hline$g$ & $-5.80 \times 10^{-11}$ & & $-7.90 \times 10^{-10}$ & & $-2.80 \times 10^{-11}$ & \\
\hline$h$ & $3.24 \times 10^{-11}$ & & $3.69 \times 10^{-11}$ & & $-1.10 \times 10^{-12}$ & \\
\hline$i$ & $-1.80 \times 10^{-13}$ & & $3.10 \times 10^{-13}$ & & $1.95 \times 10^{-15}$ & \\
\hline$j$ & $1.03 \times 10^{-13}$ & & $-8.80 \times 10^{-16}$ & & $1.02 \times 10^{-16}$ & \\
\hline
\end{tabular}

